\title{
"Trivializing" Generalizations of some Izergin-Korepin-type Determinants
}

\section{Tewodros Amdeberhan ${ }^{1}$ and Doron Zeilberger ${ }^{2}$}

${ }^{1}$ Tulane Univ., Dept of Math., New Orleans, LA 70118, USA

${ }^{2}$ Rutgers Univ., Dept of Math., Piscataway, NJ 08854, USA

received May 8, 2007, revised June 13, 2007; July 31, 2007, accepted July 31, 2007.

We generalize (and hence trivialize and routinize) numerous explicit evaluations of determinants and pfaffians due to Kuperberg, as well as a determinant of Tsuchiya. The level of generality of our statements render their proofs easy and routine, by using Dodgson condensation and/or Krattenthaler's factor exhaustion method.

All our matrices will be assumed to be embedded inside an infinite matrix.

The first theorem adds parameters to the determinant formulas found in Kuperberg [ $\mathrm{Ku}$. Theorem 15], as well as older determinants, mentioned there, due to Cauchy, Stembridge, Laksov-Lascoux-Thorup, and Tsuchiya [T]. This way, the formulation is suited to the method of [AZ]. Our proofs are much more succinct and automatable, since their generality enables an easy induction using Dodgson's rule [D, AZ], or by employing Krattenthaler's elegant factor exhaustion method [Kr1]. Relevant background for this paper can found in $[\overline{\mathrm{Ku}}]$, and references thereof.

\section{Theorem 1}

$$
\begin{gathered}
\operatorname{det}\left(\frac{1}{x_{i}+y_{j}+A x_{i} y_{j}}\right)_{i, j}^{1, n}=\frac{\prod_{i<j}\left(x_{j}-x_{i}\right)\left(y_{j}-y_{i}\right)}{\prod_{i, j}^{n}\left(x_{i}+y_{j}+A x_{i} y_{j}\right)} . \\
\operatorname{det}\left(\frac{1}{x_{i}+y_{j}}-\frac{1}{1+x_{i} y_{j}}\right)_{i, j}^{1, n}=\frac{\prod_{i<j}\left(1-x_{i} x_{j}\right)\left(1-y_{i} y_{j}\right)\left(x_{j}-x_{i}\right)\left(y_{j}-y_{i}\right)}{\prod_{i, j}^{n}\left(x_{i}+y_{j}\right)\left(1+x_{i} y_{j}\right)} \cdot \prod_{i=1}^{n}\left(1-x_{i}\right)\left(1-y_{i}\right) . \\
\operatorname{det}\left(\frac{A y_{j}+B x_{i}}{y_{j}+x_{i}}\right)_{i, j}^{1, n}=(A-B)^{n-1} \frac{\left(A \prod_{j}^{n} y_{j}+(-1)^{n-1} B \prod_{i}^{n} x_{i}\right) \prod_{i<j}\left(x_{i}-x_{j}\right)\left(y_{i}-y_{j}\right)}{\prod_{i, j}^{n}\left(x_{i}+y_{j}\right)} . \\
\operatorname{det}\left(\frac{1-x_{i} y_{j}}{y_{j}-x_{i}}\right)_{i, j}^{1, n}=(-1)^{\left(\begin{array}{c}
n \\
2
\end{array}\right)} \frac{\left(\prod_{j}^{n}\left(1+x_{j}\right)\left(1-y_{j}\right)+\prod_{i}^{n}\left(1-x_{i}\right)\left(1+y_{i}\right)\right) \prod_{i<j}\left(x_{i}-x_{j}\right)\left(y_{i}-y_{j}\right)}{2 \prod_{i, j}^{n}\left(y_{j}-x_{i}\right)} .
\end{gathered}
$$


Sketch of Proof: An automatic application of Dodgson Condensation [D, AZ].

\section{Corollary 1 (Cauchy, Stembridge, Laksov-Lascoux-Thorup)}

$$
\begin{gathered}
\operatorname{det}\left(\frac{1}{x_{i}+y_{j}}\right)_{i, j}^{1, n}=\frac{\prod_{i<j}\left(x_{j}-x_{i}\right)\left(y_{j}-y_{i}\right)}{\prod_{i, j}^{n}\left(x_{i}+y_{j}\right)} \\
\mathrm{Pf}^{2}=\operatorname{det}\left(\frac{x_{j}-x_{i}}{x_{j}+x_{i}}\right)_{i, j}^{1,2 n}=\prod_{i<j \leq 2 n} \frac{\left(x_{i}-x_{j}\right)^{2}}{\left(x_{i}+x_{j}\right)^{2}} . \\
\operatorname{Pf}^{2}=\operatorname{det}\left(\frac{x_{j}-x_{i}}{1-x_{i} x_{j}}\right)_{i, j}^{1,2 n}=\prod_{i<j \leq 2 n} \frac{\left(x_{i}-x_{j}\right)^{2}}{\left(1-x_{i} x_{j}\right)^{2}} .
\end{gathered}
$$

Remark 1 Notice that the latter two statements apply only to even-dimensional matrices. An error from $[\overline{K u}]$ in the second formula has been corrected here. Pf stands for Pfaffian of a matrix.

The next theorem generalizes, and presents variations of, several of the determinants that appear in Theorems 16 and $17[\mathrm{Ku}]$ (typos corrected in [ $\mathrm{Kr} 2$, Theorems 13 and 14]). Below, $Z_{1}, Z_{2}, Z_{3}, Z_{4}, Z_{5}$ are defined by (here $\gamma(a, b)=a-b, \tau(a, b)=a+b$ )

$$
\begin{aligned}
& Z_{1}(p, q ; x, y)_{i, j}=\frac{\gamma\left(q^{j-i}, x^{j-i}\right)}{\tau\left(p^{j-i}, y^{j-i}\right)}, \quad Z_{2}(p, q ; x, y)_{i, j}=\frac{\gamma\left(q^{1+j-i}, x^{1+j-i}\right)}{\tau\left(p^{1+j-i}, y^{1+j-i}\right)}, \\
& Z_{3}(p, q ; x, y)_{i, j}=\frac{\gamma\left(q^{-1+j-i}, x^{-1+j-i}\right)}{\tau\left(p^{-1+j-i}, y^{-1+j-i}\right)}, \quad Z_{4}(q, q ; x, x)_{i, j}=\frac{\gamma\left(q^{a+j-i}, x^{a+j-i}\right)}{\tau\left(q^{a+j-i}, x^{a+j-i}\right)}, \\
& Z_{5}(q, q ; x, x)_{i, j}=\frac{\tau\left(q^{b+j-i}, x^{b+j-i}\right)}{\gamma\left(q^{b+j-i}, x^{b+j-i}\right)},
\end{aligned}
$$

for $a \in \mathbb{Z}, b= \pm n, \pm(n+1), \ldots$ Let $\delta_{e, n}=\frac{1+(-1)^{n}}{2}$ denote Kronecker's delta function centered at the even integers, and let $\lambda_{i, j}=1$, if $i \neq j$ and $\lambda_{i, i}=0$.

Theorem 2 Write $\gamma(a, b)=a-b, \tau(a, b)=a+b$, we have the matrix determinants

$$
\begin{gathered}
\operatorname{det}\left(\frac{\gamma\left(q^{n+j-i}, x^{n+j-i}\right)}{\gamma\left(p^{n+j-i}, y^{n+j-i}\right)}\right)_{i, j}^{1, n}=(p y)^{\left(\begin{array}{l}
n \\
3
\end{array}\right)} \gamma(q, x)^{n} \frac{\prod_{j>i} \gamma\left(p^{j-i}, y^{j-i}\right)^{2} \gamma\left(q p^{j-i}, x y^{j-i}\right) \gamma\left(x p^{j-i}, q y^{j-i}\right)}{\prod_{i, j} \gamma\left(p^{n+j-i}, y^{n+j-i}\right)} \\
\quad \operatorname{det}\left(\frac{\tau\left(q^{j-i}, x^{j-i}\right)}{\tau\left(p^{j-i}, y^{j-i}\right)}\right)_{i, j}^{1, n}=\frac{\prod_{2 \mid j-i>0} \gamma\left(p^{j-i}, y^{j-i}\right)^{2} \prod_{2 \nmid j-i>0} \gamma\left(q p^{j-i}, x y^{j-i}\right) \gamma\left(x p^{j-i}, q y^{j-i}\right)}{(q x)^{\left\lfloor n^{2} / 4\right\rfloor} \prod_{j>i} \tau\left(p^{j-i}, y^{j-i}\right)^{2}} \\
\operatorname{det}\left(Z_{1}\right)_{i, j}^{1, n}=\delta_{e, n} \frac{\gamma(q, x)^{n}(p y)^{n / 2}}{(q x)^{\left\lfloor n^{2} / 4\right\rfloor}} \frac{\prod_{2 \mid j-i>0} \gamma\left(p^{j-i}, y^{j-i}\right)^{2} \gamma\left(q p^{j-i}, x y^{j-i}\right) \gamma\left(x p^{j-i}, q y^{j-i}\right)}{\prod_{j>i} \tau\left(p^{j-i}, y^{j-i}\right)^{2}}
\end{gathered}
$$




$$
\begin{gathered}
\operatorname{det}\left(Z_{2}\right)_{i, j}^{1, n}=\frac{\gamma(q, x)^{n}}{(q x)^{\left\lfloor(n-1)^{2} / 4\right\rfloor}} \frac{\prod_{2 \mid j-i>0} \gamma\left(p^{j-i}, y^{j-i}\right)^{2} \gamma\left(q p^{j-i}, x y^{j-i}\right) \gamma\left(x p^{j-i}, q y^{j-i}\right)}{\tau\left(p^{n}, y^{n}\right)^{1-\delta_{e}, n} \prod_{j>i} \tau\left(p^{j-i}, y^{j-i}\right)^{2}} \\
\operatorname{det}\left(Z_{3}\right)_{i, j}^{1, n}=\frac{(-p y)^{n} \gamma(q, x)^{n}}{(q x)^{\left\lfloor(n+1)^{2} / 4\right\rfloor}} \frac{\prod_{2 \mid j-i>0} \gamma\left(p^{j-i}, y^{j-i}\right)^{2} \gamma\left(q p^{j-i}, x y^{j-i}\right) \gamma\left(x p^{j-i}, q y^{j-i}\right)}{\tau\left(p^{n}, y^{n}\right)^{1-\delta_{e, n}} \prod_{j>i} \tau\left(p^{j-i}, y^{j-i}\right)^{2}} \\
\operatorname{det}\left(Z_{4}\right)_{i, j}^{1, n}=2^{n-1} \frac{q^{n a}+(-1)^{n} x^{n a}}{(q x)^{n(n-1)(n+1-3 a) / 6}} \frac{\prod_{j \neq i} \gamma\left(q^{|j-i|}, x^{|j-i|}\right)}{\prod_{i, j} \tau\left(q^{a+j-i}, x^{a+j-i}\right)} . \\
\operatorname{det}\left(Z_{5}\right)_{i, j}^{1, n}=(-1)^{\left(\begin{array}{l}
n \\
2
\end{array}\right)} 2^{n-1} \frac{q^{n b}+x^{n b}}{(q x)^{n(n-1)(n+1-3 b) / 6}} \frac{\prod_{j \neq i} \gamma\left(q^{|j-i|}, x^{|j-i|}\right)}{\prod_{i, j} \gamma\left(q^{b+j-i}, x^{b+j-i}\right)} . \\
\operatorname{Pf}^{2}=\operatorname{det}\left(\lambda_{i, j} \frac{\gamma\left(q^{j-i}, x^{j-i}\right) \gamma\left(r^{j-i}, z^{j-i}\right)}{\gamma\left(p^{j-i}, y^{j-i}\right)}\right)_{i, j}^{1,2 n}=\frac{(y p)^{n}}{(q x r z)^{n^{2}}} \frac{\prod_{j \neq i}^{1, n} \gamma\left(p^{|j-i|}, y^{|j-i|}\right)^{2}}{\prod_{i, j}^{1, n} \gamma\left(p^{n+j-i}, y^{n+j-i}\right)^{2}} \times \\
\prod_{i, j}^{1, n} \gamma\left(q p^{|j-i|}, x y^{|j-i|}\right) \gamma\left(x p^{|j-i|}, q y^{|j-i|}\right) \gamma\left(r p^{|j-i|}, z y^{|j-i|}\right) \gamma\left(z p^{|j-i|}, r y^{|j-i|}\right) .
\end{gathered}
$$

Sketch of Proof: Identities $Z_{4}$ and $Z_{5}$ are directly amenable to Dodgson's Condensation technique [AZ]. For the remaining assertions, use the factor exhaustion method [Kr1] (see also [Ku]): the essential idea is to compare zeros and poles on both sides of the equation at hand. We leave the straightforward details to the reader.

\section{Acknowledgments}

The authors wish to thank Christian Krattenthaler for helpful suggestions. 


\section{References}

[AZ] T. Amdeberhan, D. Zeilberger, Determinants Through the Looking Glass, Adv. Appl. Math. (Foata special issue) 2/3 27 (2001), 225-230.

[D] C.L. Dodgson, Condensation of Determinants, Proceedings of the Royal Society of London 15 (1866), 150-155.

[Kr1] C. Krattenthaler, Advanced determinant calculus, Séminaire Lotharingien Combin. 42 (1999), Art. B42q.

[Kr2] C. Krattenthaler, Advanced determinant calculus: A Complement, Linear Alg. Appl. 411 (2005), 68-166.

[Ku] G. Kuperberg, Symmetry classes of alternating-sign matrices under one roof, Ann. of Math. (2) 156 (2002), 835-866.

[T] O. Tsuchiya, Determinant formula for the six-vertex model with reflecting end, J. Math. Phys. 39 (1998), 5946-5951 